\title{
BMJ Open Effects of an internet-based cognitive behavioural therapy intervention on preventing major depressive episodes among workers: a protocol for a randomised controlled trial
}

\author{
Kotaro Imamura, ${ }^{1}$ Norito Kawakami, ${ }^{1}$ Toshi A Furukawa, ${ }^{2}$ Yutaka Matsuyama, ${ }^{3}$ \\ Akihito Shimazu, ${ }^{1}$ Kiyoto Kasai ${ }^{4}$
}

To cite: Imamura K, Kawakami N, Furukawa TA, et al. Effects of an internetbased cognitive behavioural therapy intervention on preventing major depressive episodes among workers: a protocol for a randomised controlled trial. BMJ Open 2015:5:e007590.

doi:10.1136/bmjopen-2015007590

- Prepublication history for this paper is available online. To view these files please visit the journal online (http://dx.doi.org/10.1136/ bmjopen-2015-007590).

Received 6 January 2015 Revised 6 April 2015 Accepted 11 April 2015

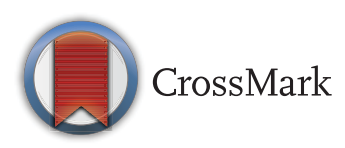

For numbered affiliations see end of article.

Correspondence to Dr Kotaro Imamura; kouima-tky@umin.ac.jp

\section{ABSTRACT}

Introduction: The aim of this study is to examine the effects of an internet-based cognitive behavioural therapy (iCBT) program on decreasing the risk of major depressive episodes (MDEs) among workers employed in a private corporate group in Japan, using a randomised controlled trial design.

Methods and analysis: All of the workers in a corporate group ( $n=20000$ ) will be recruited through an invitation email. Participants who fulfil the inclusion criteria will be randomly allocated to intervention or control groups (planned $\mathrm{N}=4050$ for each group). They will be allowed to complete the six lessons of the iCBT program within 10 weeks after the baseline survey. Those in the control group will receive the same iCBT after 12 months. The program includes several CBT skills: self-monitoring, cognitive restructuring, assertiveness, problem-solving and relaxation. The primary outcome measure is no new onset of MDE (using Diagnostic and Statistical Manual of Mental Disorders, Fourth Edition, Text Revision (DSM-IV-TR)/ DSM-5 criteria) during the 12-month follow-up. Assessment will use the web version of the WHO Composite International Diagnostic Interview V.3.0 depression section.

Ethics and dissemination: The Research Ethics Review Board of Graduate School of Medicine, the University of Tokyo (No. 3083-(2)), approved the study procedures.

Trial registration number: The study protocol is registered at the UMIN Clinical Trials Registry (UMINCTR; ID=UMIN000014146).

\section{INTRODUCTION}

Depressive disorder is one of the most prevalent psychiatric disorders, affecting around 340 million people worldwide, ${ }^{1}$ and it is associated with a substantial deterioration in quality of life and economic loss in the community and the workplace. ${ }^{2}{ }^{3}$ Thus, the primary prevention of depressive disorder is an important strategy for global mental health. In addition, stress has enormous socioeconomic implications for all spheres of employment in terms of absenteeism, staff turnover, lost productivity, poor morale, etc. ${ }^{4}$ Although the burden of stress and depression on the workplace is substantial, ${ }^{6}$ few studies aiming to prevent psychosocial problems have been conducted.

Two previous meta-analyses have shown that cognitive behaviour therapy (CBT) is an effective preventive measure for major depressive disorder. One meta-analysis reported that the risk of depressive disorder decreased by $16 \%$ on average in the intervention group and summarised 15 various types of CBTs. ${ }^{7}$ Another meta-analysis of randomised controlled trials (RCTs) with a CBT program, 'Coping with Depression (CwD)', showed that the program can also prevent major depressive disorder and indicated a $38 \%$ decrease in the risk among participants in the program. ${ }^{9}$ However, major limitations exist in the dissemination of these CBT interventions: the programs require that professionals be well trained in CBT; ${ }^{10-12}$ time, cost and stigma are other barriers to access to a CBT program. ${ }^{13}$

An innovative way to deliver CBT-based treatment widely is by using computerised CBT (CCBT) and CCBT via the internet (iCBT). Both CCBT and iCBT programs teach basic information and skills based on the same CBT principles as face-to-face CBT programs, with a highly structured format comprised of educational lessons, homework assignments and supplementary resources. ${ }^{14}$ Previous studies have shown a significant positive treatment effect of CCBT and iCBT programs on 
depression and anxiety in the clinical setting. ${ }^{15}$ An iCBT program is particularly beneficial with its high anonymity ${ }^{16}$ and high accessibility. ${ }^{17}$ Recently, there have been increasing applications of iCBT for preventing depression. Using self-reported symptoms of depression as an outcome, one study of adolescents reported a significant prevention effect of iCBT programs, though it included only male participants. ${ }^{18}$ In addition, one study of university students ${ }^{19}$ and one community-based study ${ }^{20}$ reported a significant effect of iCBT programs on improving depressive symptoms in non-clinical settings. However, one community-based study failed to show a significant effect. $^{21}$ We also have reported that a six-session iCBT program successfully improved symptoms of depression in an RCT in the workplace. ${ }^{22}$ However, a search of the literature revealed only one previous RCT (conducted by the authors), ${ }^{23}$ which investigated the effect of an iCBT program on reducing the risk of major depressive episodes (MDEs) diagnosed according to the Diagnostic and Statistical Manual of Mental Disorders (DSM)-IV diagnostic criteria. ${ }^{24}$ The control group was also provided with a treatment program during the follow-up. ${ }^{23}$ Thus, evidence for the effect of an iCBT on reducing risk of MDE is still very limited.

A previous follow-up survey of employees in a company showed that the incidence of DSM-IV major depressive disorder was $2.8 \%$ during 12 months in Japan. ${ }^{25}$ If we randomise about 8000 participants equally to intervention and control groups (4000 in each group), the total incidence will be 146 (55 and 91 in the intervention and control groups, respectively), assuming that incidence ratio $(\mathrm{IR})=0.62$ (see sample size calculation in method and analysis for details). Since the number of participants will be large, it would not be possible to conduct face-to-face or even telephone interviews to ascertain the occurrence of MDE during the follow-up; it is a feasible strategy to use the web-based self-report version of a standard structured interview, such as the WHO-Composite International Diagnostic Interview (CIDI) V.3.0 depression section, ${ }^{26}{ }^{27}$ which has been shown to have good concordance with the clinical diagnosis of $\mathrm{MDE}^{28}$ and an acceptable 1-year test-retest reproducibility. ${ }^{25}$ While a self-report assessment of MDE is clearly a major limitation, only such an instrument is feasible for a large-scale trial to reduce the risk of MDE diagnosed strictly following DSM-IV/DSM-5 criteria, which cannot be made by a symptom checklist.

\section{Objectives}

The present study is a 12-month follow-up large-scale RCT. An improved iCBT program, which was based on the results of the previous study, ${ }^{22}$ will be used. The purposes of this randomised controlled study are as follows: (1) to decrease the risk of DSM-IV/DSM-5-defined MDE through the 12-month follow-up among workers in Japan; (2) to examine the effects of the iCBT program on improving the symptoms of depression at 3-month, 6-month and 12-month follow-ups among workers who have subthreshold depressive symptoms in Japan; (3) to examine the effects of the iCBT program on improving work engagement and work performance at 3-month, 6-month and 12-month follow-ups among workers in Japan; and (4) to examine the cost-effectiveness of the iCBT program. We expect that: (1) the iCBT program will reduce the risk of MDE during the 12-month follow-up; (2) it will improve symptoms of depression at 3-month, 6-month and 12-month follow-ups among workers who have subthreshold depressive symptoms at baseline; (3) it will improve work engagement and work performance at 3-month, 6-month and 12-month followups; and (4) the program will be cost-effective.

\section{Trial design}

The study will be a two-arm, parallel-group, treatment as usual (TAU)-controlled, non-blinded randomised study. The allocation ratio of the intervention group to the control group is 1 to 1 . Participants will be randomly allocated either to the intervention group or to the control group after they have completed a baseline online questionnaire survey. Online follow-up surveys will be conducted 3, 6 and 12 months after the baseline. The study protocol was registered at the UMIN Clinical Trials Registry (UMIN-CTR; ID=UMIN000014146). This protocol manuscript was reported according to the SPIRIT guideline checklist.

\section{METHODS AND ANALYSIS \\ Participants}

Working men and women will be selected according to the following criteria:

Inclusion criteria

- Age 20-60 at study entry.

- Currently employed full-time by the business company.

- Can access the internet via a PC at home or at his/ her workplace, since the server software used in this study allows access only from a PC, but not from mobile devices such as smartphones.

Exclusion criteria

1. Non-regular or part-time employees.

2. Sick leave for 15 or more days for a physical or mental condition in the past 3 months.

3. Current treatment for a mental health problem from a mental health professional.

4. An MDE within the past month as ascertained by the web version of the Japanese WHO-CIDI V.3.0.

5. A lifetime history of bipolar disorder as ascertained by the web version of the Japanese WHO-CIDI V.3.0.

\section{Procedure}

Figure 1 shows the participant flow chart of this trial. Our previous RCT reported that $47.5 \%$ of employees who received invitation emails completed a baseline survey and $10 \%$ of them had to be excluded. ${ }^{22}$ For this study, the clinical research coordinator (CRC) will send 
Figure 1 Participant flow chart.

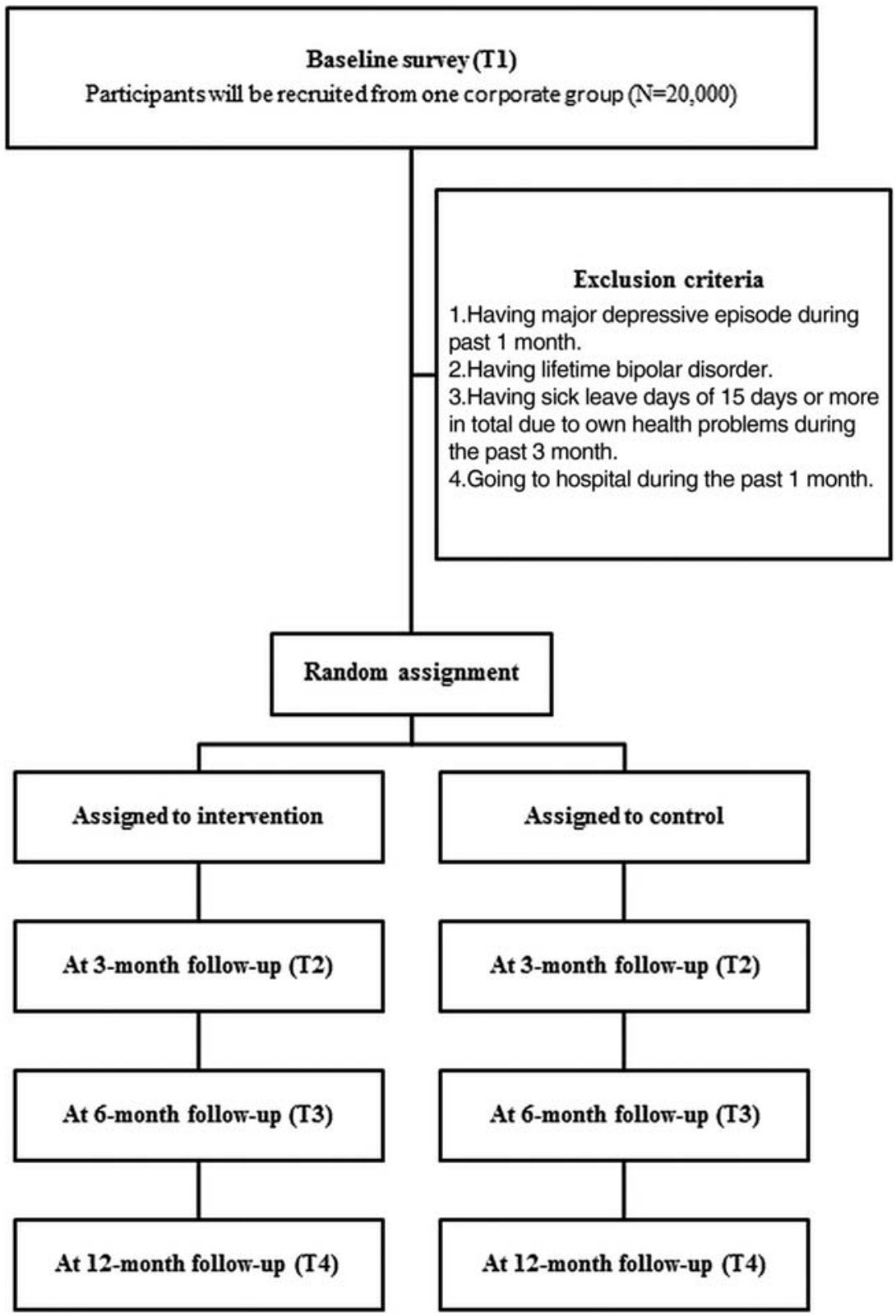

out invitations to 20000 employees, of whom 9000 are expected to give informed consent, and 8100 are expected to be eligible. These 8100 will be randomised either to the intervention group $(n=4050)$ or to the control group $(n=4050)$. They will be allowed to complete the six lessons within 10 weeks after the baseline survey. Those in the control group can receive the iCBT after 12 months. An invitation email to all workers in a corporate group will direct them to a website that includes a full explanation of the study. After reading the explanation of the study on the website, they will be asked to click on an 'agree' button to give their consent to participate in the study; then they will proceed to the baseline questionnaire page. If a candidate clicks on a 'disagree' button, the website page will close. In order for the randomisation and start of intervention to be as close as possible, the CRC will endeavour to randomise the participants immediately after closing the application and send an email with the iCBT program course description as soon as possible. A computer system for automating the randomisation of candidates on a first-come, first-served basis is not available.

\section{Intervention program}

The iCBT program called Internet CBT Program: Useful Mental Health Solutions Series for Business is a 6-week webbased training course that provides CBT-based stress management skills. ${ }^{22}$ This program is structured in six lessons, with one lesson per week. About $30 \mathrm{~min}$ are needed for each lesson, including the homework. This program can be used anywhere the internet is available.

One of the unique features of the program is that training is provided along with a Manga (Japanese comic) story of a psychologist and a client to facilitate the understanding of the participants. Several merits of using a comic story with Manga characters have been 
acknowledged in research on education in school. First, it helps motivate participants to stay in the program. ${ }^{29}$ Second, it facilitates easy learning. A program with text combined with comic stories is easier for learners to understand compared with a text-only program. ${ }^{29} 30$ Third, using a comic story fosters learners' interest in the program. ${ }^{30}$ These merits might be applicable to education in the workplace because most Japanese people of working age are familiar with comics.

The present iCBT program was developed with two established CBT packages as its basis. One is the cognitive therapy program developed by Beck. ${ }^{31}$ The other is the 'CwD' program developed by Lewinsohn. ${ }^{8}$ The CBT skill components included in the program are: selfmonitoring, cognitive restructuring, assertiveness, problem-solving and relaxation. The behavioural activation technique, a main component of the $\mathrm{CwD}$, is not included in the present RCT in order to be consistent with the previous RCT.22 23 At the end of each lesson, participants will be asked to submit homework on a voluntary basis, to receive feedback from trained staff (eg, clinical psychologists) to facilitate their understanding. Feedback will be sent to the participants within 2 days after their submission.

Table 1 shows the contents of the iCBT program. The program includes self-monitoring skills (in lesson 2), cognitive restructuring skills (in lessons 3 and 4), assertiveness skills (in lesson 5), problem-solving skills (in lesson 6) and relaxation skills (in lesson 4). In this study, the cognitive restructuring method was adopted as the primary and main cognitive approach, as it had previously been shown to be effective in reducing depression. ${ }^{32}$ Assertiveness and problem-solving training, as well as training in relaxation, were chosen as supplementary behavioural approaches to enhance the effect of the program.

\section{Lesson 1: learning about stress}

In this lesson, participants learn about a psychological stress model. ${ }^{33}$ A guiding character, clinical psychologist Miss Rino, speaks about the relationship between stressors and stress reactions. Homework in this lesson includes respondents self-checking their stressors and stress reactions to help them identify these factors/conditions.

\section{Lesson 2: knack for self-case formulation based on a CB model}

In this lesson, participants learn about a cognitive behavioural (CB) model, especially the five-part model ('five-part' refers to five areas: situation, thoughts, emotions, behaviour and physical feelings) ${ }^{34}$ and a self-case formulation based on this model. Case formulation is a method used to understand the problem of a client. ${ }^{35}$ Case formulation is necessary for clients to choose an appropriate approach to change the vicious circles of these five areas. Miss Rino introduces a five-part CB model using a vignette of a worker with a work-related problem. Homework in this lesson includes selfmonitoring using the five-part model.

\section{Lesson 3: try cognitive restructuring part 1}

In this lesson, participants learn about Beck's cognitive model and the acquired self-monitoring skills based on this model. The model postulates that an individual's mood and behaviour are affected by his/her automatic thoughts, which are shaped by dysfunctional schemas. ${ }^{36}$ The cognitive restructuring technique is one of the standard cognitive approaches of CBT utilised to change an automatic negative thought into an actual, realistic and flexible thought. ${ }^{31}$ Miss Rino gives a lecture on a cognitive ABC model (Activating/Actual event, Belief, and Consequence) ${ }^{31} 3738$ and on identifying the automatic thoughts that cause a negative mood. Homework in this lesson includes a self-monitoring exercise of participants' negative mood caused by an automatic thought in a particular situation selected by the participants.

\section{Lesson 4: try cognitive restructuring, part 2}

In this lesson, participants learn cognitive restructuring skills. Miss Rino teaches participants how to change an automatic negative thought into an actual thought. In the latter half of the lesson, participants learn a relaxation technique using a breathing method. Relaxation techniques are often added to the CBT intervention for workers, and they have shown significant effects on

\begin{tabular}{|c|c|c|}
\hline $\begin{array}{l}\text { Lesson } \\
\text { number }\end{array}$ & Title & Contents \\
\hline 1 & Learning about stress & $\begin{array}{l}\text { Learning about a psychological stress model modified for this } \\
\text { iCBT program }\end{array}$ \\
\hline 2 & $\begin{array}{l}\text { Knack for a self-case formulation based } \\
\text { on a CB model }\end{array}$ & $\begin{array}{l}\text { Learning about a CB model and how to do self-monitoring } \\
\text { based on CBT }\end{array}$ \\
\hline 3 & Try cognitive restructuring, part 1 & Learning about cognitive restructuring \\
\hline 4 & Try cognitive restructuring, part 2 & $\begin{array}{l}\text { Learning about cognitive restructuring and relaxation using a } \\
\text { breathing method }\end{array}$ \\
\hline 5 & Knack for communication & Learning about active listening and assertiveness \\
\hline 6 & How to solve your problem effectively & Learning about problem-solving methods \\
\hline
\end{tabular}


improving depression. ${ }^{39}$ Homework in this lesson includes an exercise on cognitive restructuring. On the basis of the homework of lesson 3, participants try to reconsider the rationale behind the automatic thought, seek alternative thinking and replace automatic thought with rational thinking.

\section{Lesson 5: knack for communication}

In this lesson, participants learn active listening (AL) and assertiveness skills. AL is a way of listening and responding to another person with an aim to improve mutual understanding. ${ }^{40}$ It is applied to non-therapeutic situations as a tool for better communication. Assertiveness is typically defined as the legitimate and honest expression of one's personal rights, feelings, beliefs and interests without violating or denying the rights of others. ${ }^{41}{ }^{42}$ In order to communicate assertively, the DESC (Describe, Express, Specify, and Choose or Consequence) script is used. ${ }^{43}$ Assertiveness training can help employees change their job environments by teaching them to appropriately communicate their concerns to supervisors, coworkers or subordinates. ${ }^{44}$ Assertiveness training has often been used as a supplemental component of stress management interventions in the workplace. ${ }^{45}$ In this lesson, Miss Rino also teaches $\mathrm{AL}$ and assertiveness skills based on the DESC script. Homework in this lesson includes an assertiveness exercise based on the DESC script.

\section{Lesson 6: how to solve your problem effectively}

In this lesson, participants learn a problem-solving technique based on problem-solving therapy. Problemsolving therapy is a CB intervention that focuses on training adaptive problem-solving attitudes and skills. ${ }^{46}$ A rational problem-solving style involves the deliberate and systematic application of four major problem-solving skills: (1) problem definition and formulation, (2) generation of alternative solutions, (3) decision-making and (4) solution implementation and verification. ${ }^{47}$ Problem-solving training is often used in stress management intervention in the workplace. ${ }^{39} 48$ In this lesson, Miss Rino teaches participants how to sort out the problem and make a list of solutions using problemsolving methods. Homework in this lesson includes a problem-solving exercise.

\section{Intervention group}

Participants in the intervention group will complete six weekly lessons and homework within the iCBT program. They will be allowed to complete the six lessons and submit their homework within 10 weeks after the baseline survey. The participants will be reminded by email to complete each lesson and/or submit their homework if they have not already done so. Reminders will be sent from the research office to the participants every Monday.

\section{Control group}

Participants in the control group will be able to use an internal employee assistance program service, such as consulting with a physician or a psychologist, and groupor online education/training programs for promoting mental health, as a TAU. These programs contained few descriptions of CBT knowledge and skills.

\section{Outcomes}

Table 2 shows an overview of the outcome measures. The primary outcome measure will be assessed at the baseline, and at the 6-month and 12-month follow-ups. All secondary outcomes, except for the time preference, will be assessed at the baseline, the 3-month follow-up (end of acute phase treatment), the 6-month follow-up and the 12-month follow-up. The time preference will be assessed at the baseline and the 12-month follow-up. Non-respondents will receive reminder email at least two times from the research centre for each of the follow-up surveys, at 3, 6 and 12-months.

\section{Primary outcome}

\section{Incidence of MDE}

The primary outcome measure is the onset of MDE during the 12-month follow-up. To collect accurate information of the onset of MDE, assessments will be conducted at the 6-month and 12-month follow-ups. The onset of MDE during the follow-up will be assessed using the web version of the Japanese WHO-CIDI V.3.0 depression section ${ }^{26} 27$ according to DSM, Fourth Edition, Text Revision (DSM-IV-TR) criteria. The face-to-face version of WHO-CIDI V.3.0 was translated into Japanese and proved to be valid for diagnosing MDE. ${ }^{49}$ The web version asks respondents the same set of questions and uses the skip logics of the depression section, and a diagnosis of MDE is automatically produced by a computer program using an algorithm specific to WHO-CIDI V.3.0. The web version has been shown to have a good concordance with the clinical diagnosis of $\mathrm{MDE}^{28}$ and to be reliable in a 1-year test-retest survey. ${ }^{25}$ While the WHO CIDI V.3.0 was originally designed to produce a diagnosis according to DSM-IV-TR criteria, the instrument can also produce a diagnosis of MDE based on DSM- 5 criteria. ${ }^{50}$

Since respondents will be asked to report any episode of MDE, along with the month of onset, at both the 6-month and 12-month follow-ups, there is a possibility of discrepancy in the reported onset information. In the case of such discrepancy, the reported onset at the 6-month follow-up will be used for the purposes of this study.

\section{Secondary outcomes}

\section{Beck Depression Inventory-II}

The Beck Depression Inventory II (BDI-II) is a 21-item self-report inventory that measures depressive symptoms such as sadness, pessimism, suicidal thoughts or wishes, tiredness or fatigue, loss of energy, and loss of pleasure, 
Table 2 Overview of outcome measures

\begin{tabular}{|c|c|c|c|c|c|}
\hline Measurement & Aim & $\begin{array}{l}\text { Baseline } \\
\text { (T1) }\end{array}$ & $\begin{array}{l}\text { 3-Month } \\
\text { follow-up (T2) }\end{array}$ & $\begin{array}{l}\text { 6-Month } \\
\text { follow-up (T3) }\end{array}$ & $\begin{array}{l}\text { 12-Month } \\
\text { follow-up (T4) }\end{array}$ \\
\hline $\begin{array}{l}\text { The web version of the } \\
\text { Japanese WHO-CIDI } \\
\text { V.3.0 depression section }\end{array}$ & $\begin{array}{l}\text { Duration before the onset of a } \\
\text { major depressive episode }\end{array}$ & $x$ & & $x$ & $x$ \\
\hline BDI-II & Severity of depression & $x$ & $x$ & $x$ & $x$ \\
\hline K6 & $\begin{array}{l}\text { Severity of psychological } \\
\text { distress }\end{array}$ & $x$ & $x$ & $x$ & $x$ \\
\hline $\mathrm{HPQ}$ & Work performance & $x$ & $x$ & $x$ & $x$ \\
\hline Sick leave days & $\begin{array}{l}\text { Sick leave days in the past } \\
3 \text { months }\end{array}$ & $\mathrm{x}$ & $x$ & $x$ & $x$ \\
\hline UWES & Work engagement & $x$ & $x$ & $x$ & $x$ \\
\hline Time preference & Time preference & $x$ & & & $x$ \\
\hline EQ-5D & Quality of life & $x$ & $x$ & $x$ & $x$ \\
\hline Healthcare use & Healthcare use & $x$ & $x$ & $x$ & $x$ \\
\hline
\end{tabular}

among others. ${ }^{51} 52$ Each item is scored on a scale ranging from 0 to 3 , with a higher score indicating more serious depressive symptoms.

\section{Kessler's Psychological Distress Scale}

Psychological distress will be measured by the Japanese version of Kessler's Psychological Distress Scale (K6). ${ }^{53} \mathrm{~K} 6$ consists of six items assessing the frequency with which respondents have experienced symptoms of psychological distress during the past 30 days. The response options range from 0 (none of the time) to 4 (all of the time). The internal reliability and validity found in previous studies are acceptable. ${ }^{53}$

\section{Health and Productivity Questionnaire}

The WHO Health and Productivity Questionnaire (HPQ) is a self-report instrument designed to estimate the workplace costs of health problems in terms of selfreported sickness absence (absenteeism) and reduced job performance (presenteeism). ${ }^{55}$ Previous studies have documented significant associations $(\mathrm{r}=0.61-0.87)$ of HPQ work hours assessments with payroll records ${ }^{55}$ and job performance assessments with supervisor ratings $(\mathrm{r}=0.52),{ }^{56}$ as well as other administrative records (area under the curve, 0.58-0.72). ${ }^{27}$ Respondents will be asked to rate their overall work performance during the past 4 weeks. The item will be scored on an 11-point scale ranging from 0 (worst possible performance) to 10 (best possible performance). High scores indicate a high degree of perceived work performance.

\section{Sick leave days during the past 3 months}

Respondents will be asked to report the number of sick leave days they took during the past 3 month.

\section{Utrecht Work Engagement Scale}

Work engagement will be assessed using the short form of the Japanese version of the Utrecht Work
Engagement Scale (UWES). ${ }^{57}$ The UWES consists of three subscales (ie, vigour, dedication, absorption) comprising nine items. Items are scored on a seven-point scale ranging from 0 (never) to 6 (always). Examples of items are "At my job, I feel strong and vigorous" (vigour), "I am enthusiastic about my job" (dedication) and "I am immersed in my work" (absorption). A total score is calculated from all nine items.

\section{Time preference (time discounting)}

Time preference (time discounting) is one's relative valuation for having something (eg, money) currently compared with its valuation at a later date. It may moderate the effect of an iCBT program. Among others, ${ }^{58}$ in this study, time preference is assessed by the following procedure ${ }^{5960}$ The respondents will be asked to choose between two options, A and $\mathrm{B}$. The respondent receives 1 million yen (around US\$12 000) in a month when he/ she chooses option A, while he/she receives a different amount in 13 months when he/she chooses option B. This question consists of nine choices (see table 3). For example, for the sixth choice, the respondents compare 1 million yen today to 1020000 yen (around US\$12 240) in 13 months. In this case, choosing option $\mathrm{B}$ instead of option $\mathrm{A}$ is the same as receiving a $2 \%$ annual increase. The questionnaire is shown in table 3 , where the amount received under option A is specified as 1 million yen and the imputed interest rate for option B changes from $-5 \%$ to over $10 \%$.

\section{Quality of life}

Health-related quality of life will be assessed with the EQ-5D. ${ }^{61}$ The EQ-5D consists of five items covering five dimensions (mobility, self-care, usual activities, pain/discomfort and anxiety/depression), each of which is rated as causing 'no problems' to 'unable to', and a visual analogue scale. It is a widely applied quality of life 
Table 3 Questionnaire to elicit the time-discount rate

\begin{tabular}{llll}
\hline $\begin{array}{l}\text { Option A (receipt } \\
\text { in a month) }\end{array}$ & $\begin{array}{l}\text { Option B (receipt } \\
\text { in 13 months) }\end{array}$ & $\begin{array}{l}\text { Interest rate } \\
\text { (annual), \% }\end{array}$ & Circle A or B \\
\hline 1 million yen & 950000 yen & -5 & $\mathrm{~A}$ \\
1 million yen & 1 million yen & 0 & $\mathrm{~A}$ \\
1 million yen & 1001000 yen & 0.10 & $\mathrm{~A}$ \\
1 million yen & 1005000 yen & 0.50 & $\mathrm{~A}$ \\
1 million yen & 1010000 yen & 1 & $\mathrm{~A}$ \\
1 million yen & 1020000 yen & 2 & $\mathrm{~A}$ \\
1 million yen & 1060000 yen & 6 & $\mathrm{~B}$ \\
1 million yen & 1100000 yen & 10 & $\mathrm{~B}$ \\
1 million yen & Over 1100 000 yen & Over 10 & $\mathrm{~B}$ \\
\hline
\end{tabular}

Suppose you have two mutually exclusive options to receive some money. You may choose option ' $A$ ' to receive 1 million yen in a month; or option ' $\mathrm{B}$ ' to receive a different amount in 13 months. Compare the amounts and delay until its receipt in option ' $A$ ' with option ' $B$ ' and indicate which option you would prefer for each pair of all nine choice pairs.

instrument, and its reliability and validity are well established. $^{61}$

\section{Key economic outcomes \\ Clinical end points}

A cost-effectiveness analysis will be based on the main outcome of depression-free years gained. Depressionfree years will be assessed by calculating the difference in follow-up lengths and the duration of any MDE (ie, period of time in weeks that a person meets DSM-IV criteria). In the cost-utility analysis, quality-adjusted life years (QALYs) will be the clinical end point. QALYs will be obtained from the EQ-5D.

\section{Costs}

Direct medical costs will be estimated from the questions about healthcare use. Indirect costs stemming from production losses due to absenteeism and presenteeism will be assessed with the WHO HPQ ${ }^{55}$

\section{Healthcare use}

Respondents will be asked to report on their healthcare use at any time during the past 3 months as follows: (1) consultation with a general practitioner (if yes, the number of times); (2) history and number of hospitalisations (if yes, the number of days of hospitalisation); (3) use of medication of any kind; and (4) use of consultation with an industrial physician or the employee assistance program (if yes, the number of times).

\section{Sample size calculation}

A meta-analysis of CBT interventions using the ' $\mathrm{CwD}$ ' program reported that the average effect size (IR) for prevention of MDE was 0.62 (95\% CI 0.43 to 0.91 ) at post-test. ${ }^{9}$ A follow-up survey of employees in a company showed that the incidence of major depressive disorder was $2.8 \%$ during 12 months. ${ }^{25}$ We applied a method proposed by Rubinstein $e t a l^{62}$ to calculate a minimal sample size and a statistical power for a proportional hazard model analysis. Thus, if we equally randomise 8272 to an intervention group and control group (4136 participants in each group), we will have $90 \%$ power to detect a treatment effect, assuming that IR $=0.62$. However, these calculations ignore dropout. We expect that $75 \%$ will complete our 12-month follow-up, resulting in 3102 respondents in each group at 12 months. In this situation, we will have $80 \%$ power to detect the IR of 0.62 .

On the other hand, a previous systematic review has shown that the incidence of major depressive disorder was greater in participants with subthreshold depressive symptoms than in participants without subthreshold depressive symptoms. ${ }^{63}$ By stratifying participants according to $\mathrm{K} 6$, we will conduct subgroup analyses targeting the high-risk group.

\section{Randomisation}

Participants who fulfil the inclusion criteria will be randomly allocated to intervention or control groups. Stratified permuted-block randomisation will be conducted as well. Participants will be stratified into 16 strata according to two factors: K6 score ( 5 or greater or less than 5) in the baseline survey and the eight workplaces to which they belong. The intervention effect may vary according to the severity of psychological distress at baseline. In addition to the analysis of the whole sample (to examine the universal intervention effect), we will also analyse data by a priori-defined subgroups (to examine the selective intervention effect). A stratified permutedblock random table will be generated by an independent biostatistician. Enrolment will be conducted by a CRC, and assignment will be conducted by an independent research assistant. The stratified permuted-block random table will be password protected and blinded to the researcher. Only the research assistant will be able to access it during the work of random allocation.

\section{Statistical methods}

\section{Clinical efficacy}

A survival analysis will be conducted to test for the effectiveness of the intervention by comparing the survival time not having an MDE between the intervention and control groups. The survival time of each 
participant was calculated as months from baseline to the onset of MDE or the termination of the observation. The length of follow-up for each participant will be represented by either the number of months between the baseline and the onset of MDE or the end of the 12-month follow-up period (6-month follow-up if a respondent dropped out at the 12-month follow-up), whichever comes first. The cumulative incidence of MDE at the 6-month and 12-month follow-ups as well as event-free survivals in every follow-up month will be estimated by the Kaplan-Meier method, and the statistical significance of the difference between the cumulative proportions of having an MDE at the 6-month and 12-month follow-ups in the intervention and control groups will be tested. A log-rank test will be conducted to test the difference in the survival probabilities between the intervention and control groups. A single covariate Cox discrete time hazard model will be used to test the difference and to estimate the HR and the $95 \%$ CIs of having an MDE in the intervention group compared with the control group. The intervention effect will also be estimated, adjusting for dependent censoring using the inverse probability of censoring weighted method for conducting a sensitivity analysis. ${ }^{64}$ A similar analysis will also be conducted using an alternative case definition, such as having a moderate level of depression (a BDI-II score of 20 or above).

For secondary outcomes (ie, symptoms of depression), a mixed model for repeated measures conditional growth model analysis will be conducted using a group (intervention and control)* time (baseline, 3-month, 6-month and 12-month follow-ups) interaction as an indicator of intervention effect. An intention-to-treat analysis will be conducted as well, using the mixed model for repeated measures conditional growth model analysis. Effect sizes and 95\% CIs will be calculated using Cohen's $d$ among those who completed the questionnaire at baseline and at a follow-up. The values of $0.2,0.5$ and 0.8 are generally interpreted as being suggestive of small, medium and large effects, respectively. ${ }^{65}$ In addition, the number needed to treat to reduce depressive symptoms or psychological distress to achieve improvement from subthreshold depression will be calculated. Referencing the cut-off scores of BDI-II and K6 of previous studies, ${ }^{51}{ }^{66}$ all statistical analyses will be conducted using the SPSS Statistics V.21.0.

\section{Subgroup analysis}

The effectiveness of the program may differ according to the initial severity of depression. We will therefore use, as one stratification factor, high/low subthreshold depression (ie, participants who scored 5 or more in K6) at the baseline survey and analyse the results according to a priori-defined subgroups (selective intervention effect).

\section{Economic analysis}

In the cost-effectiveness analysis, the incremental costeffectiveness ratio (ICER) will be stated as costs per depression-free months gained, whereas the ICER in the cost-utility analysis will represent the costs per qualityadjusted life month gained. Bootstrapping will be used to test the robustness of the ICERs and to quantify the uncertainty around the ratios that will be graphically represented on a cost-effectiveness plane.

\section{Data monitoring}

A Data and Safety Monitoring Board (DSMB) will be set up, including an independent chair and at least two independent members. The DSMB will meet every 3 months after the first client is randomised. The purpose of the meetings will be to review the report prepared by the CRC. The CRC will prepare DSMB reports to monitor recruitment progress and data collection (eg, percentage completing each follow-up).

\section{ETHICS AND DISSEMINATION \\ Ethical and safety considerations}

We have prepared a website that contains a full explanation of the study. Before the baseline survey, participants will be invited to read the explanation on the website and asked to click on an 'agree' button to show their consent to participate in the study. They will then proceed to a baseline questionnaire page. Candidates will be fully informed that their participation is totally voluntary, that even after voluntarily participating they can withdraw from the study without stating the reason, and that neither participation nor withdrawal will cause any advantage or disadvantage to them. Written consent is not required by the Ethical Guidelines for Biomedical Research Involving Human Subjects, Japan; the Research Ethics Review Board of Graduate School of Medicine/ Faculty of Medicine, the University of Tokyo has approved this procedure to obtain the participants' consent.

\section{Data confidentiality}

The survey data will be temporarily stored on a server placed at the Department of Mental Health, Graduate School of Medicine, the University of Tokyo. After the survey, the collected data will be moved to a password-locked stand-alone PC. The collected data will be stored as linkable anonymising data. The data will be accessible only by the CRC.

\section{Dissemination of research findings}

The main findings of this study will be disseminated via publications in peer-reviewed international journals. Presentations of study findings will also be offered at relevant research conferences, and local academic symposia and seminars.

\section{Strengths and limitations}

The greatest strength of this study design is its focus on the effect of the iCBT program on preventing the onset of MDE using a large-scale RCT design in a healthy working population. This study is also intended to add 
evidence for the effect of CBT programs on positive health outcomes (eg, work engagement and work performance) and economic evaluation of iCBT on the primary prevention strategy among healthy workers.

One of the major weaknesses of this study is that an MDE will be measured by self-report, which may be affected by the perception of the participants or by situational factors at work. The validity of the web-based CIDI depression section has been established partially but needs further clarification and refinement. The other limitation is that the participants will be recruited from one corporate group in Japan. Most of them have their own PCs in their offices or homes. The participants may also be assumed to have experience with using a PC and studying in online programs. Therefore, generalisation of the findings to populations that do not share the characteristics of the participants may be limited.

\author{
Author affiliations \\ ${ }^{1}$ Department of Mental Health, Graduate School of Medicine, The University of \\ Tokyo, Tokyo, Japan \\ ${ }^{2}$ Departments of Health Promotion and Human Behavior and of Clinical \\ Epidemiology, Graduate School of Medicine/School of Public Health, Kyoto \\ University, Kyoto, Japan \\ ${ }^{3}$ Department of Biostatistics, Graduate School of Medicine, The University of \\ Tokyo, Tokyo, Japan \\ ${ }^{4}$ Department of Neuropsychiatry, Graduate School of Medicine, The University \\ of Tokyo, Tokyo, Japan
}

Acknowledgements The authors appreciate the help of the following persons in completing this project: Takayuki Narumi, Jun Naoi, Keisuke Kito, Chinatsu Narumi, Hayato Mori, Sayaka Horii, Mitsuyasu Mizusaki, Chihiro Hoshino, Kie Fujii, Aya Matsumoto and Izumi Unezawa. They are also grateful to Dr Takashi Fukuda for his advice of economic evaluation.

Contributors KI, NK, TAF, YM and KK conceived and designed the experiments. $\mathrm{KI}$ and NK contributed reagents/materials/analysis tools. $\mathrm{KI}$, NK, TAF and AS wrote the paper. All authors read and approved the final paper.

Funding This study is supported by the Grant-in-Aid for Young Scientists (B) 2014 (grant number 26860433) from the Japan Society for the Promotion of Science.

Competing interests $\mathrm{KI}$ is employed part-time by the Chugai Pharmaceutical Company and Medical Care Toranomon as a clinical psychologist. NK has received lecture fees from Meiji, Otsuka, EAP Consulting, Fujitsu Software Technologies, Japan Productivity Center, Occupational Health Foundation, Japan Housing Finance Agency, Aishin-Seiki and Japan Dental Association, and consultancy fees from Sekisui Chemicals, Junpukai Health Care Center and the Osaka Chamber of Commerce and Industry. He has received royalties from Igaku-Shoin, Taishu-kan, Nanko-do, Nanzan-do, PHP Publication and Fujitsu Software Technologies, Ltd, and research grants from The Japanese Ministry of Education, Science, and Technology, the Japanese Ministry of Health, Labor and Welfare, Fujitsu Software Technologies, Ltd, Softbank, Co, Ltd and the Japan Management Association. TAF has received lecture fees from Eli Lilly, Meiji, Mochida, MSD, Pfizer and Tanabe-Mitsubishi, and consultancy fees from Sekisui and Takeda Science Foundation. He is a diplomate of the Academy of Cognitive Therapy. He has received royalties from Igaku-Shoin, Seiwa-Shoten and Nihon Bunka Kagaku-sha. The Japanese Ministry of Education, Science, and Technology, the Japanese Ministry of Health, Labor and Welfare, and the Japan Foundation for Neuroscience and Mental Health have funded his research projects. YM has received lecture fees from the Union of Japanese Scientists and Engineers, EPS Co, Ltd, and statcom Co, Ltd, and consultancy fees from Zeria pharmaceutical Co, Ltd, Ono pharmaceutical Co, Ltd and Mebix Co, Ltd. He has received royalties from Igaku-Shoin and Ewanami-Shoten. AS works for Hitachi Systems, Ltd as a part-time consultant. He is on the advisory board for Junpukai Health Care Center and Ds's Mental Health Labo. He has received royalties from Baifukan, Kawashima-shoten, Seishin-shobou and Seiwa-Shoten. KK has received lecture fees from Astellas, Novartis, Eli Lilly, Otsuka, Dainippon-Sumitomo and Yoshitomi pharmaceutical companies. He has received collaborative research grants from Astellas, Hitachi Co and Hitachi Medical, and research grants from Yoshitomi, Dainippon-Sumitomo, Astellas and GSK.

Ethics approval The Research Ethics Review Board of Graduate School of Medicine/Faculty of Medicine, the University of Tokyo approved the study procedures (no 3083-(2)).

Provenance and peer review Not commissioned; externally peer reviewed.

Open Access This is an Open Access article distributed in accordance with the Creative Commons Attribution Non Commercial (CC BY-NC 4.0) license, which permits others to distribute, remix, adapt, build upon this work noncommercially, and license their derivative works on different terms, provided the original work is properly cited and the use is non-commercial. See: http:// creativecommons.org/licenses/by-nc/4.0/

\section{REFERENCES}

1. Hosman C, Jané Llopis E, Saxena S. Prevention of mental disorders: effective interventions and policy options: summary report Geneva: World Health Organization, 2004

2. Kessler RC. The costs of depression. Psychiatr Clin North Am 2012;35:1-14

3. Saarni SI, Suvisaari J, Sintonen H, et al. Impact of psychiatric disorders on health-related quality of life: general population survey. Br J Psychiatry 2007;190:326-32.

4. Grime PR. Computerized cognitive behavioural therapy at work: a randomized controlled trial in employees with recent stress-related absenteeism. Occup Med 2004;54:353-9.

5. Noblet A, Lamontagne AD. The role of workplace health promotion in addressing job stress. Health Promot Int 2006;21:346-53.

6. Billings DW, Cook RF, Hendrickson A, et al. A web-based approach to managing stress and mood disorders in the workforce. J Occup Environ Med 2008;50:960-8.

7. Cuijpers $P$, van Straten A, Smit F, et al. Preventing the onset of depressive disorders: a meta-analytic review of psychologica interventions. Am J Psychiatry 2008;165:1272-80.

8. Lewinsohn PM. The Coping with depression course: a psychoeducational intervention for unipolar depression. Eugene, OR: Castalia Pub. Co., 1984.

9. Cuijpers P, Munoz RF, Clarke GN, et al. Psychoeducational treatment and prevention of depression: the "Coping with Depression" course thirty years later. Clin Psychol Rev 2009;29:449-58.

10. World Health Organization. Department of Mental Health and Substance Abuse. Atlas: Mental Health Resources in the World 2001. Geneva: World Health Organization, 2001.

11. World Health Organization. Department of Mental Health and Substance Abuse. Mental health atlas 2005. Rev ed. Geneva: World Health Organization, 2005.

12. World Health Organization. Department of Mental Health and Substance Abuse. Mental health atlas 2011. Updated and revised ed. Geneva: World Health Organization, 2011.

13. Ruwaard J, Lange A, Bouwman M, et al. E-mailed standardized cognitive behavioural treatment of work-related stress: a randomized controlled trial. Cogn Behav Ther 2007;36:179-92.

14. Titov N, Andrews G, Sachdev P. Computer-delivered cognitive behavioural therapy: effective and getting ready for dissemination. F1000 Med Rep 2010;2:49.

15. Andrews G, Cuijpers P, Craske MG, et al. Computer therapy for the anxiety and depressive disorders is effective, acceptable and practical health care: a meta-analysis. PLOS ONE 2010;5:e13196.

16. Gega L, Marks I, Mataix-Cols D. Computer-aided CBT self-help for anxiety and depressive disorders: experience of a London clinic and future directions. J Clin Psychol 2004;60:147-57.

17. Spek V, Cuijpers $P$, Nyklicek I, et al. Internet-based cognitive behaviour therapy for symptoms of depression and anxiety: a meta-analysis. Psychol Med 2007;37:319-28.

18. Calear AL, Christensen H, Mackinnon A, et al. The YouthMood Project: a cluster randomized controlled trial of an online cognitive behavioral program with adolescents. J Consult Clin Psychol 2009;77:1021-32

19. Lintvedt OK, Griffiths KM, Sorensen K, et al. Evaluating the effectiveness and efficacy of unguided internet-based self-help 
intervention for the prevention of depression: a randomized controlled trial. Clin Psychol Psychother 2013;20:10-27.

20. Christensen H, Griffiths KM, Jorm AF. Delivering interventions for depression by using the internet: randomised controlled trial. BMJ 2004;328:265-68A.

21. Patten SB. Prevention of depressive symptoms through the use of distance technologies. Psychiatr Serv 2003;54:396-8.

22. Imamura K, Kawakami N, Furukawa TA, et al. Effects of an internet-based cognitive behavioral therapy (iCBT) program in Manga format on improving subthreshold depressive symptoms among healthy workers: a randomized controlled trial. PLOS ONE 2014;9:e97167.

23. Imamura K, Kawakami N, Furukawa T, et al. Does Internet-based cognitive behavioral therapy (iCBT) prevent major depressive episode for workers? A 12-month follow-up of a randomized controlled trial. Psychol Med 2015:1-11. Published Online Fist 7 Jan 2015. doi:10.1017/S0033291714003006

24. American Psychiatric Association. Diagnostic and statistical manual of mental disorders. DSM-IV-TRß: American Psychiatric Pub, 2000.

25. Shimoda $\mathrm{H}$, Inoue $\mathrm{A}$, Tsuno $\mathrm{K}$, et al. One-year test-retest reliability of a Japanese web-based version of the WHO Composite International Diagnostic Interview (CIDI) for major depression in a working population. Int J Methods Psychiatr Res 2014. Published Online First: 21 Nov 2014. doi:10.1002/mpr.1437

26. Kawakami N, Takeshima T, Ono Y, et al. Twelve-month prevalence, severity, and treatment of common mental disorders in communities in Japan: preliminary finding from the World Mental Health Japan Survey 2002-2003. Psychiatry Clin Neurosci 2005;59:441-52.

27. Kessler RC, Ustun TB. The World Mental Health (WMH) Survey Initiative Version of the World Health Organization (WHO) Composite International Diagnostic Interview (CIDI). Int J Methods Psychiatr Res 2004;13:93-121.

28. Inoue A, Kawakami N, Tsuno K, et al. Organizational justice and major depressive episodes in Japanese employees: a crosssectional study. J Occup Health 2013;55:47-55.

29. Hutchinson $\mathrm{KH}$. An experiment in the use of comics as instructional material. J Educ Sociol 1949;23:236-45.

30. Sones WWD. The comics and instructional method. J Educ Sociol 1944;18:232-40.

31. Beck AT. Cognitive therapy of depression. New York: Guilford Press, 1979.

32. Jakobsen JC, Hansen JL, Storebø OJ, et al. The effects of cognitive therapy versus 'no intervention' for major depressive disorder. PLOS ONE 2011;6:e28299.

33. Lazarus RS. Emotion and adaptation. New York: Oxford University Press, 1991.

34. Padesky CA, Greenberger D. Clinician's guide to mind over mood New York: Guilford Press, 1995.

35. Adams H. Further clarifications of case formulation. Behav Ther 1996;19:78-78.

36. Beck AT. Depression: clinical, experimental, and theoretical aspects New York: Hoeber Medical Division, 1967.

37. Ellis A. Humanistic psychotherapy: the rational-emotive approach. New York: Julian Press, 1973.

38. Ellis A, Harper RA. A new guide to rational living. Englewood Cliffs, NJ: Prentice-Hall, 1975.

39. Richardson KM, Rothstein HR. Effects of occupational stress management intervention programs: a meta-analysis. J Occup Health Psychol 2008;13:69-93.

40. Rogers CR. The necessary and sufficient conditions of therapeutic personality-change. J Consult Psychol 1957;21:95-103.

41. Alberti RE. Differentiating assertion and aggression-some behavioral guidelines-reply. Behav Ther 1977;8:353-4.

42. Alberti RE, Emmons ML. Your perfect right: assertiveness and equality in your life and relationships. 8th edn. Atascadero, CA Impact Publishers, 2001.

43. Bower SA, Bower GH. Asserting yourself: a practical guide for positive change. Updated ed. Cambridge, MA: Da Capo Life Long, 2004.

44. Schwartz GE. Stress Management in occupational settings. Public Health Rep 1980;95:99-108.
45. Bellarosa C, Chen PY. The effectiveness and practicality of occupational stress management interventions: a survey of subject matter expert opinions. J Occup Health Psychol 1997;2:247-62.

46. Bell AC, D'Zurilla TJ. Problem-solving therapy for depression: a meta-analysis. Clin Psychol Rev 2009;29:348-53.

47. D'Zurilla TJ, Goldfried MR. Problem solving and behavior modification. J Abnorm Psychol 1971;78:107-26.

48. Bhui KS, Dinos S, Stansfeld SA, et al. A synthesis of the evidence for managing stress at work: a review of the reviews reporting on anxiety, depression, and absenteeism. J Environ Public Health 2012;2012:1-21.

49. Kawakami N, Takeshima T, Ono Y, et al. Twelve-month prevalence, severity, and treatment of common mental disorders in communities in Japan: the World Mental Health Japan 2002-2004 survey. In Kessler RC, Ustun TB, eds. The WHO World Mental Health Surveys: global perspectives on the epidemiology of mental disorders. New York, NY: Cambridge University Press, 2008:474-85.

50. Association AP. Diagnostic and statistical manual of mental disorders, (DSM-5尺). American Psychiatric Pub, 2013.

51. Beck AT, Steer RA, Brown GK. BDI-II, Beck depression inventory: manual. 2nd edn. San Antonio, TX: Psychological Corp.; Harcourt Brace, 1996

52. Hiroe T, Kojima M, Yamamoto I, et al. Gradations of clinical severity and sensitivity to change assessed with the Beck Depression Inventory-II in Japanese patients with depression. Psychiatry Res 2005;135:229-35

53. Furukawa TA, Kawakami N, Saitoh M, et al. The performance of the Japanese version of the K6 and K10 in the World Mental Health Survey Japan. Int J Methods Psychiatr Res 2008;17:152-8.

54. Kessler RC, Andrews G, Colpe LJ, et al. Short screening scales to monitor population prevalences and trends in non-specific psychological distress. Psychol Med 2002;32:959-76.

55. Kessler RC, Barber C, Beck A, et al. The world health organization health and work performance questionnaire (HPQ). J Occup Environ Med 2003;45:156-74

56. Kessler RC, Ames M, Hymel PA, et al. Using the World Health Organization Health and Work Performance Questionnaire (HPQ) to evaluate the indirect workplace costs of illness. J Occup Environ Med 2004;46:S23-37.

57. Shimazu A, Schaufeli WB, Kosugi S, et al. Work engagement in Japan: validation of the Japanese version of the Utrecht Work Engagement Scale. Appl Psychol 2008;57:510-23.

58. van der Pol M, Cairns J. Methods for eliciting time preferences over future health events. In: Scott A, Maynard A, Elliott R, eds. Advances in health economics. Wiley, 2003:41-58. http://dx.doi.org/ 10.1002/0470867922.ch3

59. Kang MI, Ikeda S. Time discounting and smoking behavior: evidence from a panel survey*. Health Econ, 2013.

60. Takada M, Kondo N, Hashimoto $H$. Japanese study on stratification, health, income, and neighborhood: study protocol and profiles of participants. J Epidemiol 2014;24:334-44.

61. Herdman M, Gudex C, Lloyd A, et al. Development and preliminary testing of the new five-level version of EQ-5D (EQ-5D-5L). Qual Life Res 2011;20:1727-36.

62. Rubinstein LV, Gail MH, Santner TJ. Planning the duration of a comparative clinical trial with loss to follow-up and a period of continued observation. J Chronic Dis 1981;34:469-79.

63. Cuijpers $P$, Smit F. Subthreshold depression as a risk indicator for major depressive disorder: a systematic review of prospective studies. Acta Psychiatr Scand 2004;109:325-31.

64. Robins JM, Finkelstein DM. Correcting for noncompliance and dependent censoring in an AIDS clinical trial with Inverse Probability of Censoring Weighted (IPCW) Log-Rank Tests. Biometrics 2000;56:779-88.

65. Cohen J. A power primer. Psychol Bull 1992;112:155-9.

66. Sakurai K, Nishi A, Kondo K, et al. Screening performance of $\mathrm{K} 6 / \mathrm{K} 10$ and other screening instruments for mood and anxiety disorders in Japan. Psychiatry Clin Neurosci 2011;65:434-41. 